\title{
Through the Looking Glass: "Remission" in Rheumatoid Arthritis
}

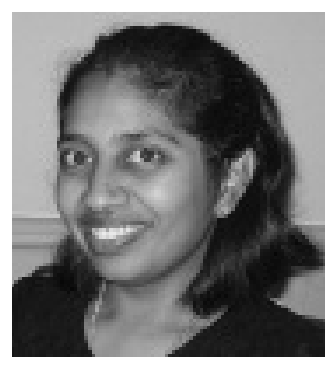

"When I use a word, it means just what I choose it to mean"

$$
\text { - Lewis Carroll: Through the Looking Glass }
$$

The original definition of rheumatoid arthritis (RA) remission dates from the 1950s when Short and Bauer described a state where "the disease was inactive, the patients were asymptomatic, and examination of the joints was negative except for residual deformity"1,2. About 3 decades later, this overall concept of remission in RA remained remarkably similar when Pinals and colleagues defined "complete" RA remission as the "total absence of all articular and extraarticular inflammation and immunological activities related to RA"3. Only a few patients with RA were fortunate enough to meet these elusive definitions, but the increasing effectiveness of disease modifying antirheumatic drugs (DMARD) and biologic agents, which were previously unavailable, now offers tangible expectations for improvement. Now, almost 6 decades later, "remission" is touted as the goal for RA treatment.

In this issue of The Journal, Ma and colleagues describe a systematic review of the literature of remission in early RA, and showcase the necessity for standardization of RA remission definitions so that direct comparisons across studies can be made 4 . The overall purpose of their extensive examination of the literature from 1996 to 2008 was to evaluate: (1) frequency of remission as defined by the American College of Rheumatology (ACR) Remission Criteria and Disease Activity Score (DAS), as well as their current modifications; (2) frequency of remission in patients given monotherapy versus those given combination therapy; and (3) effects of remission on progression of radiographic damage, in both randomized clinical trials (RCT) and prospective observational studies. Twenty RCT and 17 observational early RA studies were ultimately included in the final analyses. About $27 \%$ of 4762 RA patients with disease duration $<3$ years achieved remission criteria by either ACR or
DAS remission definitions in the observational studies, with $31 \%$ of the 4290 RA patients in the RCT achieving "remission." Patient followup ranged widely, from 6 months to 10 years. The bottom line was that, overall, remission based on DAS criteria was achieved in fewer than one-third of studied patients, while remission based on the more stringent definition (ACR remission criteria) was achieved in far fewer patients (observational studies 17\% and $15 \%-22 \%$ in RCT). Ma, et al reported only studies that utilized ACR/DAS remission measures; however, accounting for all the modifications of ACR and DAS remission measures, there were actually 6 different "remission" measures reported within the 37 studies (ACR remission criteria; modified ACR (mACR) remission excluding fatigue; mACR remission excluding morning stiffness; mACR changing the threshold of pain visual analog scale; also DAS cutpoint 1.6 and 2.6).

Among observational studies examined, only 8 provided information to calculate ACR remission rates (6/8 studies used modified ACR remission criteria), with an average rate of $17 \%$. Nine studies utilized DAS remission cutpoints with an average remission rate of $33 \%$. Across the board, DAS remission rates are about 2 times higher than the ACR remission rates. These comparisons demonstrate that DAS criteria are somewhat less stringent. This realization is not new. Makinen and colleagues' evaluation of 161 RA patients demonstrated that a substantial number can have tender and swollen joints and still be considered to be in DAS remission ${ }^{5}$, thus supporting the argument that remission as defined by DAS (and other definitions) is a reflection of low disease activity in RA, rather than a complete absence of disease.

The metaanalysis by Ma, et al also confirms that modern RA therapies improve the condition of patients, although the effects are as not large as we wish. Comparison of rates of ACR and DAS remission between DMARD monotherapy and combination therapy showed that the overall rates

See Remission in Early RA, page 1444

Personal non-commercial use only. The Journal of Rheumatology Copyright $@$ 2010 . All rights reserved. 
were quite similar [ACR remission of $15 \%$ for monotherapy (mono) vs $22 \%$ for combination (combo) therapy, DAS remission of $31 \%$ for mono vs $36 \%$ for combo]. They further investigated the random effects odds ratio (OR) for the ACR and DAS remission rates in RCT of combination therapy versus monotherapy, yielding OR of 1.69 (ACR remission) and 2.01 (DAS remission), respectively.

Radiographic outcomes for patients meeting ACR or DAS remission were demonstrated by 4 observational studies. One study showed that $45 \%$ of patients meeting ACR remission criteria developed new or increased erosions. In 2 studies comparing patients meeting DAS remission criteria versus those not meeting criteria, similar rates of radiographic progression were observed (27\% in DAS remission vs $34 \%$ not; and $54 \%$ in DAS remission vs $58 \%$ not). Overall, Ma and colleagues show there is a continuum of radiographic progression that appears to be unrelated to meeting current remission definitions. Recently, more sensitive imaging modalities such as ultrasound (US) and magnetic resonance imaging (MRI) have been used to assess inflammation in patients meeting current remission criteria. Brown and colleagues performed MRI and US on 107 RA patients deemed to be in remission (based on rheumatologist assessment, ACR remission, or DAS28/ESR remission) and found a majority of patients continued to have evidence of inflammation by both US and MRI (96\% with synovitis, $46 \%$ bone marrow edema on MRI, and $73 \%$ synovial hypertrophy, $43 \%$ increased power Doppler) ${ }^{6}$. Thus, data in the literature regarding both longitudinal progression of radiographic joint damage and MRI/US imaging of soft tissue inflammation strongly suggest that achievement of clinical remission may not be sufficient to define the absence of disease activity.

Ma and colleagues' metaanalysis highlights the heterogeneity of studies for evaluating remission in early RA with respect to differences in clinical trial/observational study design, treatment strategy, duration of sustained remission, duration of followup time, definitions of radiographic progression, and definitions of remission by ACR/DAS.

The progress in RA treatment over the last 30 years is clearly impressive and should not be minimized. Clinical trial design, biologic agents available to treat RA, and basic science successes have advanced the ability of therapeutic management to improve clinical and radiographic outcomes in RA. Perhaps the overenthusiasm about this laudable progress has engendered a belief that we can readily achieve the still elusive goal of "remission" that should be tempered. Varying definitions of "remission," ranging from lenient to very stringent, and their utilization without standardization in studies causes confusion regarding clinical expectations.

Clarity in the definition of outcome measures in RA is key. I propose that we return to basic definitions "cure" and "remission," and insist on uniformity of these definitions in RA studies. "Cure" in RA is not possible with our currently available RA therapies. I define "cure" as the permanent cessation of the underlying disease process, with the patient no longer requiring medications to treat the disease; this is akin to oncology colleagues' definition in cancer. "Cure" is understandably difficult when we don't really know the etiology of the disease, thus stressing the importance of diligent work in basic science to answer this question. Clearly, we do not yet have the capability to achieve "remission" in RA, in the strictest sense of the word, that is, cessation of the inflammatory disease process, without progression or residual evidence of inflammation seen on imaging, while continuing medications for treatment of RA. The distinction between "cure" and "remission" is the need for continued therapeutic management. In addition, there should be a time component to each: a specified time period required in these states (such as 6 months, 1 year, or 5 years) prior to deeming a patient to be in remission or cured. Remission must be a continually evolving term, incorporating scientific advances in areas of biomarkers, imaging, and other avenues of technologic advancement. I propose that we do not use the word "remission" until we are capable of truly achieving this disease state in our patients. In the meantime, we need to insist on some uniformity of reported criteria for "reduced disease activity." On the other hand, we should not let semantics get in the way of optimal treatment.

The development of both ACR and EULAR response criteria required a rigorous process, and currently academic institutions, industry, and practicing rheumatologists are largely clear about their meaning. However, discussion of remission as an outcome measure in RA is fraught with ambiguity, allowing each study to create its own definition to suit its needs and desires.

In arriving at a definition, several issues require additional attention: Should the definition of remission factor in the duration of sustained remission? Is imaging with radiographs, ultrasound, or MRI necessary to determine whether a patient is in true remission? How do we define a patient as being in drug-free remission? Lastly, is remission currently an achievable target? Clearly there is no consensus on the definition of remission so we must be very cautious in our interpretations of results of our studies. It is agreed that an achievable "therapeutic target" for optimal management of RA is needed, but a misleading definition of "remission" is not the solution. Undoubtedly, with the advancement of RA therapy, this therapeutic target will change. At a future date we may truly be capable of achieving "remission" in our patients, just not now.

VEENA K. RANGANATH, $\mathrm{MD}, \mathrm{MS}$, School of Medicine, University of California Los Angeles, Los Angeles, California, USA

Address correspondence to Dr. V. Ranganath, Rehabilitation Building, Room 32-98, UCLA, 1000 Veteran Avenue, Los Angeles, CA 90024. E-mail:vranganath@mednet.ucla.edu

Personal non-commercial use only. The Journal of Rheumatology Copyright ( 2010 . All rights reserved. 
I genuinely thank Janet Elashoff, PhD, Jennifer Grossman, $M D$, and Harold E. Paulus, MD, for their editorial help.

\section{REFERENCES}

1. Short CL, Bauer W. The course of rheumatoid arthritis in patients receiving simple medical and orthopedic measures. N Engl J Med 1948;238:142-8.

2. Short CL, Bauer W, Reynolds WE. Rheumatoid arthritis. Cambridge: Harvard University Press; 1957.

3. Pinals RS, Masi AT, Larsen RA. Preliminary criteria for clinical remission in rheumatoid arthritis. Arthritis Rheum 1981; 24:1308-15.
4. Ma MHY, Scott IC, Kingsley GH, Scott DL. Remission in early rheumatoid arthritis. J Rheumatol 2010;37:1444-53.

5. Makinen H, Kautiainen H, Hannonen P, Sokka T. Is DAS28 an appropriate tool to assess remission in rheumatoid arthritis? Ann Rheum Dis 2005;64:1410-3. Epub 2005 Jun 7.

6. Brown AK, Quinn MA, Karim Z, Conaghan PG, Peterfy CG, Hensor E, et al. Presence of significant synovitis in rheumatoid arthritis patients with disease-modifying antirheumatic drug-induced clinical remission: evidence from an imaging study may explain structural progression. Arthritis Rheum 2006; 54:3761-73.

J Rheumatol 2010;37:1371-3; doi:10.3899/jrheum.100495 